\title{
Bioavailability of Orally Administered Des-Aspartate-Angiotensin $I$ in Human Subjects
}

\author{
Kok-Onn Lee ${ }^{1}$ Edmund Feng Tian' ${ }^{2}$ Martin Hui Cai ${ }^{2} \cdot \mathrm{Hong} \mathrm{Wang}^{3,5}$. \\ Yiong-Huak $\mathrm{Chan}^{4} \cdot$ Meng-Kwoon $\mathrm{Sim}^{5,6}$
}

Published online: 22 November 2017

(c) The Author(s) 2017. This article is an open access publication

\begin{abstract}
In an earlier single-dose escalation study to evaluate the safety and pharmacokinetics of orally administered des-aspartate-angiotensin I (DAA-I) in healthy subjects, the plasma level of DAA-I could not be determined because DAA-I is rapidly degraded in the circulation. The present study investigated the oral bioavailability of DAA-I by measuring the prostaglandin $E_{2}$ metabolite (PGEM) in the plasma samples of the same trial. PGEM is a stable derivative of $\mathrm{PGE}_{2}$, which has been shown to be a biomarker of DAA-I. The data show that plasma from two of the three subjects who were orally administered the efficacious preclinical dose of $0.70 \mathrm{mg} / \mathrm{kg}$ DAA-I exhibited a significant PGEM peak at 5-6 h postdose. Plasma of subjects who were administered 0.08 and $1.5 \mathrm{mg} / \mathrm{kg}$ DAA-I, the subefficacious and two-times efficacious dose, respectively, did not exhibit a similar PGEM
\end{abstract}

Meng-Kwoon Sim

simmk@alumni.nus.edu.sg

1 Department of Medicine, National University of Singapore, 1E Kent Ridge Road, NUHS Tower Block Level 10, Singapore 119228, Singapore

2 School of Applied Science, Temasek Polytechnic, 21 Tampines Avenue 1, Singapore 529757, Singapore

3 Singapore Nuclear Research and Safety Initiative, National University of Singapore, 1 CREATE Way, \#04-01 CREATE Tower, Singapore 138602, Singapore

4 Biostatistics Unit, Yong Loo Lin School of Medicine, National University Health System, 1E Kent Ridge Road, NUHS Tower Block Level 11, Singapore 119228, Singapore

5 Department of Pharmacology, Yong Loo Lin School of Medicine, Block MD 3 Level 4 \#04-01, 16 Medical Drive, Singapore 117600, Singapore

6 SimPharma, 60 Paya Lebar Road \#12-55, Paya Lebar Square, Singapore 409051, Singapore peak. This observation is concordant with the known in vivo actions of DAA-I, especially its hypoglycemic action where maximum efficacy occurred at a dose of $0.7 \mathrm{mg} / \mathrm{kg}$, and decreased to nil at the two-times efficacious dose. The onset of the PGEM peak at 5-6 h postdose was closed to the 4-h onset of absorption of $\left[\mathrm{C}^{14}\right]$ DAA-I seen in preclinical rat studies, albeit the absorption kinetics between rodents and humans are not identical. The occurrence of polymorphism of enzymes involved in the formation and degradation of $\mathrm{PGE}_{2}$ is common, and this has been attributed to contributing to the variation in response, onset and peak PGEM observed among the three subjects who were administered the efficacious dose.

\section{Key Points}

The bioavailability of orally administered desaspartate-angiotensin I (DAA-I) was investigated in human subjects.

Prostaglandin $\mathrm{E}_{2}$ metabolite (PGEM), a stable metabolite derivative of $\mathrm{PGE}_{2}$ was measured as a biomarker of DAA-I in plasma samples obtained from 15 subjects in a single-dose phase I trial on DAA-I.

Plasma of two of the three subjects who were administered a preclinical efficacious dose of DAA-I $(0.7 \mathrm{mg} / \mathrm{kg})$ showed a significant PGEM peak concentration at 5-6 h postdose. No similar significant PGEM peak was present in the plasma of the other subjects who were administered a subefficacious and a two-time efficacious dose. This observation was in concordance with the known in vivo actions of DAA-I. 


\section{Introduction}

Des-aspartate-angiotensin I (DAA-I) is an endogenous angiotensin peptide that has been shown to exhibit efficacy in animal models of 11 human pathologies [1-3]. DAA-I acts as an agonist on the angiotensin $\mathrm{AT}_{1}$ receptor and releases prostaglandins, which mediate its actions $[1,4]$. In cultured human umbilical vein endothelial cells (HUVECs), DAA-I specifically increased the production of prostaglandin $\mathrm{E}_{2}\left(\mathrm{PGE}_{2}\right)$ and prostaglandin $\mathrm{I}_{2}\left(\mathrm{PGI}_{2}\right)$ via cyclooxygenase (COX)-1 at sub-nanomolar concentrations, but did not stimulate production of these two prostaglandins via COX-2 [4]. DAA-I was rapidly degraded by circulating enzymes when infused into the systemic circulation of the dog [5, 6] and human [7]. Despite being an endogenous peptide and having a rapid systemic metabolism, DAA-I has been shown to be active when administered orally $[1-3]$. This is probably attributed to its ability to cross Caco-2 cells via passive diffusion [8], indicating that it would cross intestinal epithelial cells, and its activity at concentrations lower than the $\mathrm{Km}$ of enzymes [1]. However in a single-dose phase I study, where subjects were orally administered a subefficacious, efficacious, and twice efficacious dose in terms of its preclinical hypoglycaemic activity, we found no significant increase in subjects' plasma DAA-I concentration over a period of $12 \mathrm{~h}$ post-administration [9]. We have advanced various possibilities to explain the absence of DAA-I bioavailability in the phase I study. In a further attempt to obtain physical evidence to show that DAA-I is orally bioavailable, and support its oral efficacy, we measured PGEM, a stable derivative of $\mathrm{PGE}_{2}$ metabolites, as a biomarker in the plasma samples of the single-dose phase I study. The assay was carried out using a commercial ELISA kit, as preliminary experiments showed that the limit of detection for $\mathrm{PGE}_{2}$ and its metabolites, as determined by liquid chromatography-tandem mass spectrometry (LC-MS/MS), was higher than their basal levels in the plasma samples. The results show that two of the three subjects administered the efficacious dose exhibited a plasma PGEM profile, with a maximum at 5-6 h post-administration. No similar profile was seen in the other plasma samples of subjects administered the other two doses of DAA-I.

\section{Materials and Methods}

\subsection{Plasma Samples}

Plasma samples were from subjects of a single-dose phase I trial on DAA-I [9]. The samples had been stored frozen at $80{ }^{\circ} \mathrm{C}$ for a period of 18 months, prior to analysis. Three cohorts of six subjects were recruited for the phase I trial and the collected plasma samples were labelled A1-A6, B1-B6, and $\mathrm{C} 1-\mathrm{C} 6$ for Cohorts $\mathrm{A}, \mathrm{B}$, and $\mathrm{C}$, respectively. Two subjects in each cohort were administered placebo (sterile water), and the other four subjects from Cohorts A, B and C were administered $0.08,0.7$ and $1.5 \mathrm{mg} / \mathrm{kg}$ DAA-I in an aqueous solution, respectively. Volunteers were randomly selected into treatment cohorts. The placebo group plasma samples are denoted with A3, A5, B2, B6, C2 and C5. Due to the use of a plasma sample from each cohort for preliminary analysis in earlier studies, only plasma samples from five subjects in each cohort were available for the present study.

\subsection{Plasma Sampling Time}

For each subject, plasma samples were taken at 10 different time points, i.e. at time 0 (predosing), and $15 \mathrm{~min}, 30 \mathrm{~min}$, $1 \mathrm{~h}, 2 \mathrm{~h}, 3 \mathrm{~h}, 4 \mathrm{~h}, 5 \mathrm{~h}, 6 \mathrm{~h}$ and $12 \mathrm{~h}$ postdosing.

\subsection{Assay of Prostaglandin $\mathbf{E}_{2}$}

$\mathrm{PGE}_{2}$ in the plasma samples was assayed as its stable end metabolite, PGEM, using a Cayman Prostaglandin E Metabolite ELISA Kit (Cat No. 514531; Cayman Chemical, Ann Arbor, MI, USA). The analysis was performed according to the manufacturer's instructions, as described previously [3]. The Cayman's assay is based on the competition between a stable PGE metabolite derivative, PGEM, and a PGEM-acetylcholinesterase conjugate (PGEM tracer) for a limited number of PGEM-specific rabbit anti-serum binding sites. $\mathrm{PGE}_{2}$ metabolites in plasma were first converted to PGEM. Briefly, each $100 \mu \mathrm{L}$ plasma sample was incubated overnight at $37^{\circ} \mathrm{C}$ with $30 \mu \mathrm{L}$ of $1 \mathrm{M}$ carbonate buffer. $40 \mu \mathrm{L}$ of $1 \mathrm{M}$ phosphate buffer and $30 \mu \mathrm{L}$ of ELISA buffer were added to the plasma mixture. With this treatment, the plasma sample was diluted twice. Eight $100 \mu \mathrm{L}$ aliquots of PGEM ELISA standard, each aliquot containing an increasing concentration of PGEM, were similarly treated. A $50 \mu \mathrm{L}$ aliquot of each treated plasma sample was incubated in a well of an assay plate precoated with goat anti-rabbit immunoglobulin G. $50 \mu \mathrm{L}$ PGEM tracer and $50 \mu \mathrm{L}$ PGEM ELISA antiserum were added into each well. After an 18-h incubation at ambient temperature, the plate was rinsed five times with wash buffer. An aliquot of $200 \mu \mathrm{L}$ freshly prepared Ellman's reagent was added to each well and the plate was immediately wrapped in aluminum foil and incubated for $90 \mathrm{~min}$ in an orbital shaker. Absorbance at $412 \mathrm{~nm}$ was read after incubation. The content of PGEM in the eight standard solutions was similarly assayed. Preliminary experiments showed that readings obtained from twicediluted plasma samples fell within the $20-80 \%$ range of the 
standard curves, and provided the rationale for a two times dilution of the plasma samples.

\subsection{Statistical Analysis}

One-way analysis of variance was used to test for significance of the increase in PGEM concentrations that occurred between 4.5 and $5.5 \mathrm{~h}$ post-administration in plasma samples of subjects B4 and B5 of cohort B. Post hoc analysis was corrected using Tukey's honest significant difference (HSD) test to correct for the type I error on the pairwise comparisons. Statistical significance was set at $p<0.05$.

\section{Results}

Figure 1 displays the PGEM concentration profile in the 15 plasma samples obtained from subjects who participated in the single-dose phase I trial on DAA-I. The plasma PGEM concentration profile in two of the three samples in cohort B (B4 and B5) had a peak at 5 and 6 h post-administration, respectively. The mean concentration between 4.5 and $5.5 \mathrm{~h}$ of the two peak concentrations was significantly higher than the corresponding concentrations in the placebo samples. Additionally, the mean concentration for sample B5 was significantly higher than the corresponding concentrations of the other two samples from DAA-Itreated subjects. The period between 4.5 and $5.5 \mathrm{~h}$ postadministration covers the half hour before and half hour after the peak PGEM concentration of sample B4, and the mean plasma PGEM concentrations of cohort B during this period were used for statistical comparison. This period was selected because the peak PGEM concentration of sample B4 was lower than that of sample B5. The PGEM concentration profiles in the plasma samples of cohorts A and $\mathrm{C}$ did not exhibit a similar peak concentration.

\section{Discussion}

As therapeutics, peptides are recognized for being highly selective, efficacious, and well-tolerated [10]; however, most endogenous peptides are not suitable for use as therapeutics per se because they have a short circulating half-life. Glucagon-like peptide 1, amylin, and somatostatin are examples, and their respective analogues such as exenatide, pramlinitide and octreotide have considerable longer half-life and are used as parental drugs in lieu of the parent peptides. DAA-I, although an endogenous peptide with a similar short circulating half-life, has been shown to be active when administered orally [1-3]. Its bioavailability was estimated to be 0.06 in a study where both intravenous and oral DAA-I were shown to produce similar
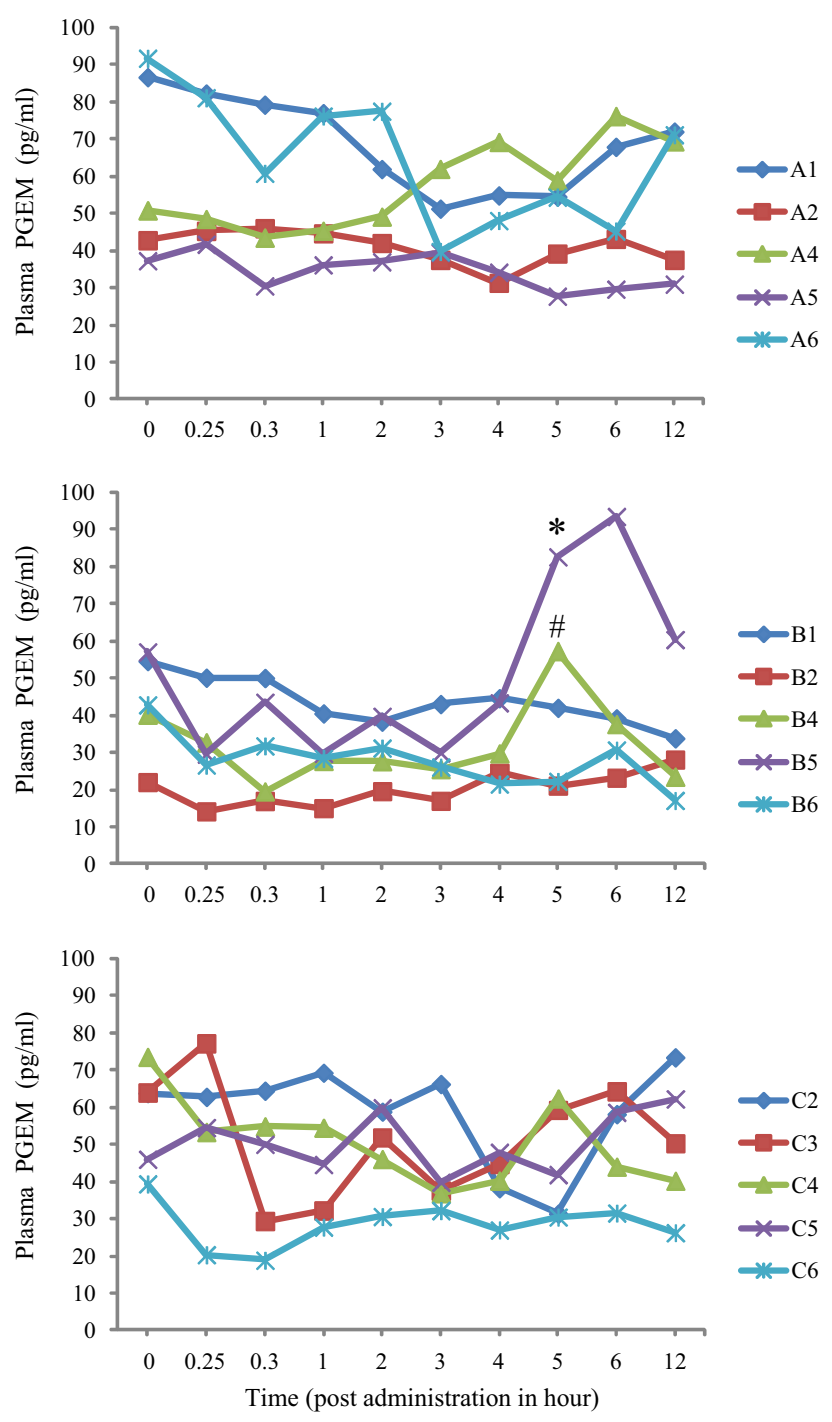

Fig. 1 Plasma PGEM concentrations. A5, B2, B6, C2 and C5 were placebo samples, and samples in the upper, middle and lower plots were from subjects who were administered $0.08,0.70$, and $1.5 \mathrm{mg} / \mathrm{kg}$ DAA-I, respectively. * Indicates significantly greater than the corresponding values of B1, B2, B4 and B6 $(p<0.01),{ }^{\#}$ indicates significantly greater than the corresponding values of B2 and B6 $(p<0.01)$

magnitudes of attenuation of cardiac hypertrophy [1]. In preclinical toxicology studies, a $\left[{ }^{14}\right]$ C-DAA-I-like peak was detected at $4 \mathrm{~h}$ after $\left[{ }^{14}\right] \mathrm{C}$-DAA-I was orally administered to rats, indicating that the onset of DAA-I absorption was $4 \mathrm{~h}$ post-administration (unpublished preclinical toxicological data). These findings, together with the ability of DAA-I to passage Caco- 2 cells via passive diffusion [8], are indications of its oral bioavailability.

The data in Fig. 1 lend further credence to the oral bioavailability of DAA-I. The bioavailability was denoted by the presence of a peak concentration of PGEM, a biomarker of DAA-I, appearing at 5-6 h post-administration in two of the three plasma samples of subjects who were 
administered the preclinical efficacious dose of $0.7 \mathrm{mg} / \mathrm{kg}$. This observation is in concordance with several known actions of DAA-I. First, DAA-I has been shown to specifically release $\mathrm{PGE}_{2}$ from cultured HUVECs [4]. This enables $\mathrm{PGE}_{2}$ to be a candidate biomarker of DAA-I as the endothelium is the likely tissue of first contact with absorbed DAA-I. HUVECs lack the enzymes that degrade $\mathrm{PGE}_{2}$, but, in systemic circulation, $\mathrm{PGE}_{2}$ is rapidly degraded to its 13,14-dihydro-15-keto metabolites, and in this study was measured as PGEM, the stable derivative of these metabolites [11]. The second commonality is the dose-dependent action of DAA-I, where doses higher than its maximum efficacious dose caused an absence of effect, as shown by the absence of peak plasma concentrations of PGEM in the plasma samples of subjects who were administered a subefficacious (cohort A) and two-times efficacious (cohort $\mathrm{C}$ ) dose. This dose-related action is also seen in the hypoglycaemic action of DAA-I in the KKAy mouse model of type 2 diabetes, where an efficacy occurred at a dose of $0.7 \mathrm{mg} / \mathrm{kg}$ DAA-I; doubling of this dose caused a total loss of the hypoglycaemic effects [12].

The PGEM peak occurred at 5-6 h post-administration in subjects B4 and B5, respectively. This is $1-2 \mathrm{~h}$ later than the $4 \mathrm{~h}$ absorption onset seen in preclinical studies, and could be due to a variation in absorption kinetics between rodents and humans, or the time taken for the released $\mathrm{PGE}_{2}$ to degrade to its 13,14-dihydro-15-keto metabolites. Variation in response to DAA-I could also have occurred among the DAA-I-treated subjects of cohort B, where subject B1 did not respond, while subject B5 had a more marked response but a later onset than subject B4. The formation of $\mathrm{PGE}_{2}$ involves multiple enzymes [13] and its breakdown to 13,14-dihydro-15-keto metabolites involves both enzymatic and non-enzymatic degradation [11]. The key enzymes involved in the multistep pathway of $\mathrm{PGE}_{2}$ formation and degradation are polymorphic $[14,15]$, and this could be responsible for the variable responses seen in the three DAA-I-treated subjects of cohort B.

\section{Conclusion}

The data in the present study are further evidence of the oral bioavailability of DAA-I. Although the bioavailability was determined by measuring PGEM, a stable metabolite derivative of the biomarker $\mathrm{PGE}_{2}$, the observed plasma PGEM profiles were in concordance with the known in vivo actions of DAA-I. This singular matching of plasma kinetics and in vivo actions is a reflection of the specific and unique properties of DAA-I.

Acknowledgements The study was supported by Grant R-184-000237-511 from the National Medical Research Council, Singapore

\section{Compliance with Ethical Standards}

Conflict of interest Kok-Onn Lee, Edmund Feng Tian, Martin Hui Cai, Hong Wang, Yiong-Huak Chan and Meng-Kwoon Sim have no conflicts of interest with regard to financial sponsorship or governmental guidelines on the publication of this article.

Open Access This article is distributed under the terms of the Creative Commons Attribution-NonCommercial 4.0 International License (http://creativecommons.org/licenses/by-nc/4.0/), which permits any noncommercial use, distribution, and reproduction in any medium, provided you give appropriate credit to the original author(s) and the source, provide a link to the Creative Commons license, and indicate if changes were made.

\section{References}

1. Sim MK. Des-aspartate-angiotensin I, a novel angiotensin $\mathrm{AT}_{1}$ receptor drug. Eur J Pharmacol. 2015;760:36-1.

2. Sim MK. The use of des-aspartate-angiotensin I in inflammation related pathologies and diseases. Patent Cooperation Treaty, International Application No. PCT/SG2011/000204, 8 Jun 2011.

3. Wang H, Sethi G, Loke WK, Sim MK. Des-aspartate-angiotensin I attenuates mortality of mice exposed to gamma radiation via a novel mechanism of action. PLoS One. 2015;10(9):e0138009.

4. Wen Q, Lee KO, Sim SZ, Xu XG, Sim MK. Des-aspartate-angiotensin I causes specific release of PGE2 and PGI2 in HUVEC via the angiotensin $\mathrm{AT}_{1}$ receptor and biased agonism. Eur $\mathrm{J}$ Pharmacol. 2015;768:173-81.

5. Gayes RP, Szidon JP, Opari S. In vivo and in vitro conversion of des-1-Asp angiotensin I to angiotensin III. Biochem Pharmacol. 1978;27:2871-7.

6. Sexton JM, Britton SL, Beierwaltes WH, Fiksen-Olsen MJ, Romero JC. Formation of angiotensin III from [des-Asp1] angiotensin I in the mesenteric vasculature. Am J Physiol Heart Circ Physiol. 1979;237:H218-23.

7. Kono T, Ikeda F, Oseko F, Imura H. Biological activity of desasp1-angiotensin I in man. J Clin Endocrinol Metab. 1980;50:40-5.

8. Chua HL, Jois S, Sim MK, Go ML. Transport of angiotensin peptides across the Caco-2 monolayer. Peptides. 2004;25:1327-38.

9. Lee KO, Khoo CM, Chowbay B, Chan YH, Sim MK. A single dose-escalation study to evaluate the safety and pharmacokinetics of orally administered des-aspartate angiotensin $\mathrm{I}$ in healthy subjects. Drugs R D. 2016;16:317-26.

10. Fosgerau K, Hoffmann T. Peptides therapeutics: current status and future directions. Drug Discov Today. 2015;20:122-8.

11. Prostaglandin E Metabolite ELISA Kit Item No 51453. Kit Booklet. https://www.caymanchem.com/pdfs/514531.pdf.

12. Sim MK, Xu XG, Wong YC, Sim SZ, Lee KO. Des-aspartateangiotensin I exerts hypoglycemic action via glucose transporter4 translocation in type 2 diabetic KKAy mice and GK rats. Endocrinology. 2007;148:5925-32.

13. Park JY, Pillinger MH, Abramson SB. Prostaglandin E2 synthesis and secretion: the role of PGE2 synthases. Clin Immunol. 2006;119:229-40.

14. Ulrich CM, Bigler J, Sparks R, Whitton J, Sibert JG, Goode EL, et al. Polymorphisms in PTGS1 $(=\mathrm{COX}-1)$ and risk of colorectal polyps. Cancer Epidemiol Biomark Prev. 2004;13:889-93.

15. Pereira C, Queirós S, Galaghar A, Sousa H, Pimentel-Nunes P, Brandao $\mathrm{C}$, et al. Genetic variability in key genes in prostaglandin $\mathrm{E}_{2}$ pathway (COX-2, HPGD, ABCC4 and SLCO2A1) and their involvement in colorectal cancer development. PLoS One. 2014;9(4):e92000. 